\title{
Cardiac Tamponade as a Manifestation of Large Cell Carcinoma of the Lung
}

Rosalind C. Watman, D.O., Arnold Conrad, M.D., Norman Riegel, M.D., Faroque Khan, M.D. Stony Brook, New York.

\begin{abstract}
The following illustrates a case study of a 38 year-old male with large cell carcinoma of the lung who subsequently developed cardiac tamponade. The incidence, as well as the association of large cell carcinoma and cardiac tamponade are discussed. Also, discussed are the pathophysiology, diagnosis and treatment of cardiac tamponade.
\end{abstract}

Key Words: Large cell carcinoma, lung carcinoma, cardiac tamponade.

DOI: http://dx.doi.org/10.5915/20-4-13301

Lung cancer is presently the leading cause of cancer death in both men and women. In 1988, it is estimated that approximately 93,000 males and 46,000 females in the United States will die of lung cancer alone. ${ }^{1}$ This tumor frequently metastasizes to the heart, with the pericardium the most common site of cardiac involvement.

This metastatic involvement can sometimes present as an emergent situation. The following case demonstrates such an example.

\section{Case report}

The patient is a 38-year-old, white male with a history of large-cell (anaplastic type) carcinoma of the lung diagnosed in January 1987, by supraclavicular lymph node biopsy, after presenting with three months of anorexia, weakness and weight loss.

The patient received local radiation therapy (total of 5,000 rads) over the course of 10 days and was subsequently treated with one dose of chemotherapy consisting of Velban, methotrexate, cis-platinum and mitomycin.

Ten days after receiving chemotherapy he was readmitted to a local hospital presenting with acute onset of shortness of breath. A chest x-ray revealed a left upper lobe mass and a globular-shaped heart.

From the Department of Medicine

Nassau County Medical Center

State University of New York

Reprint requests: Rosalind C. Watman, D.O.

Nassau County Medical Center

2201, Hempstead Turnpike

East Meadow, New York 11554
Echocardiogram confirmed the presence of a large pericardial effusion, and the patient was subsequently transferred to Nassau County Medical Center for further evaluation and treatment.

Upon arrival to our hospital, the patient was noted to be markedly short of breath and unable to lie flat. Physical examination was significant for a blood pressure of $120 / 70$, with a paradoxical pulse of 20 $\mathrm{mmHg}$. Examination of the neck revealed jugular venous distention at a $45^{\circ}$ angle. Distant heart sounds were noted on cardiac examination. Examination of the extremities revealed clubbing of the fingers. Chest x-ray demonstrated a left upper lobe mass with well-defined margins measuring $8 \mathrm{~cm}$ in diameter, an enlarged cardiac silhouette and a small left pleural effusion (Figure 1). Sinus tachycardia, diminished QRS voltage, and nonspecific ST-T wave changes were noted on the admission EKG (Fgure 2). Admission labs were remarkable for a decreased $\mathrm{Hgb}$ and Hct $(9 \mathrm{gm} / \mathrm{dl}$ and $27 \%)$ with normal indices, decreased platelet count $\left(23,500 / \mathrm{mm}^{3}\right)$, decreased WBC $\left(2,400 / \mathrm{mm}^{3}\right)$ and a prolonged PT $(20.2 / 11.4$ seconds) and PTT (38.2/30.9 second). Arterial blood gases on admission were; $\mathrm{pH}=7.58, \mathrm{PO}_{2}=52$, $\mathrm{PCO}_{2}=19$.

An echocardiogram revealed a large anterior and posterior pericardial effusion with diastolic collapse of the right atrium (Figure 3).

The patient was immediately transfused with 10 units of platelets and 4 units of fresh frozen plasma and underwent emergency pericardiocentesis which yielded $1500 \mathrm{cc}$ of sanguinous fluid. Cytological examination revealed mesothelial atypia probably reactive Pap Class II-III.

Following the procedure, a dramatic improvement in the patient's symptoms, along with resolution of 


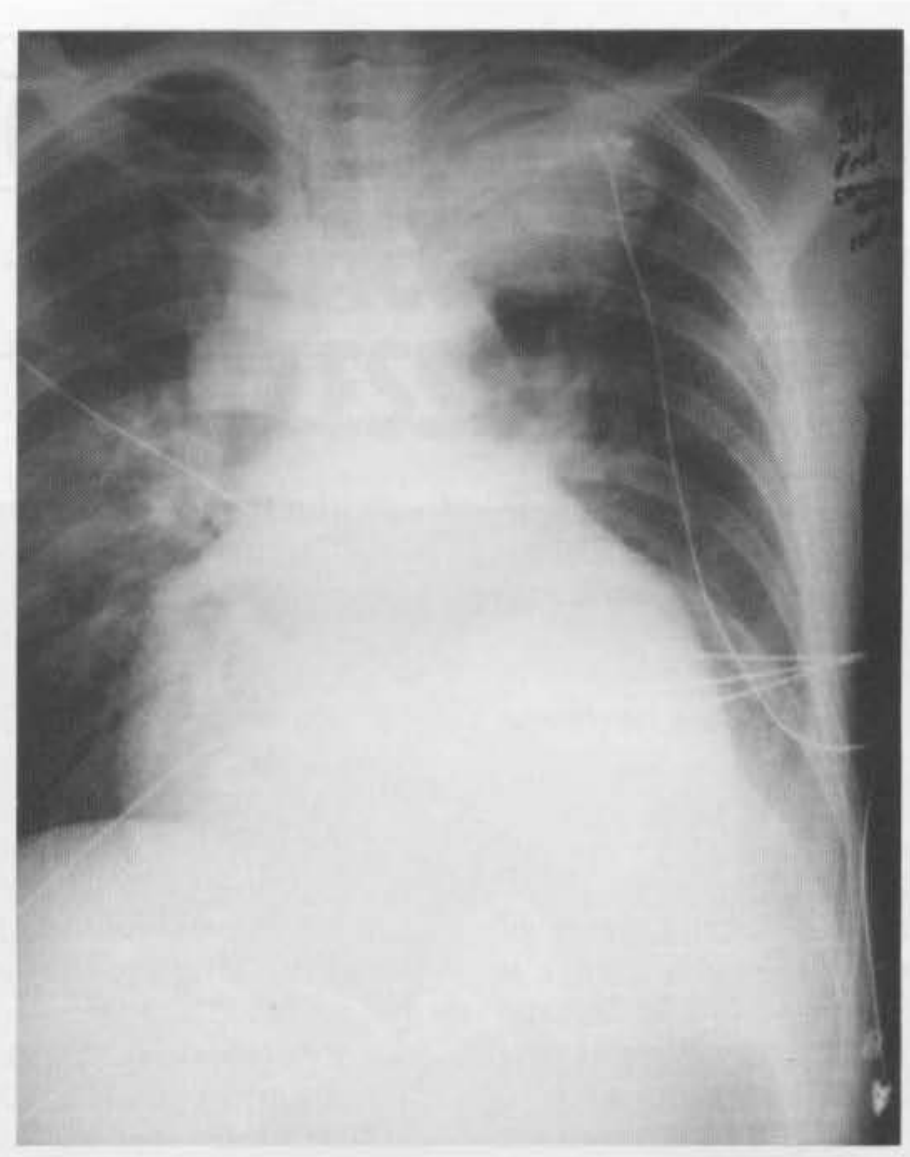

Figure 1. Chest X-ray AP view revealing large cardiac silhouette, central pulmonary arteries obscured by pericardial fluid, probably left pleural effusion, and a left upper lobe mass.

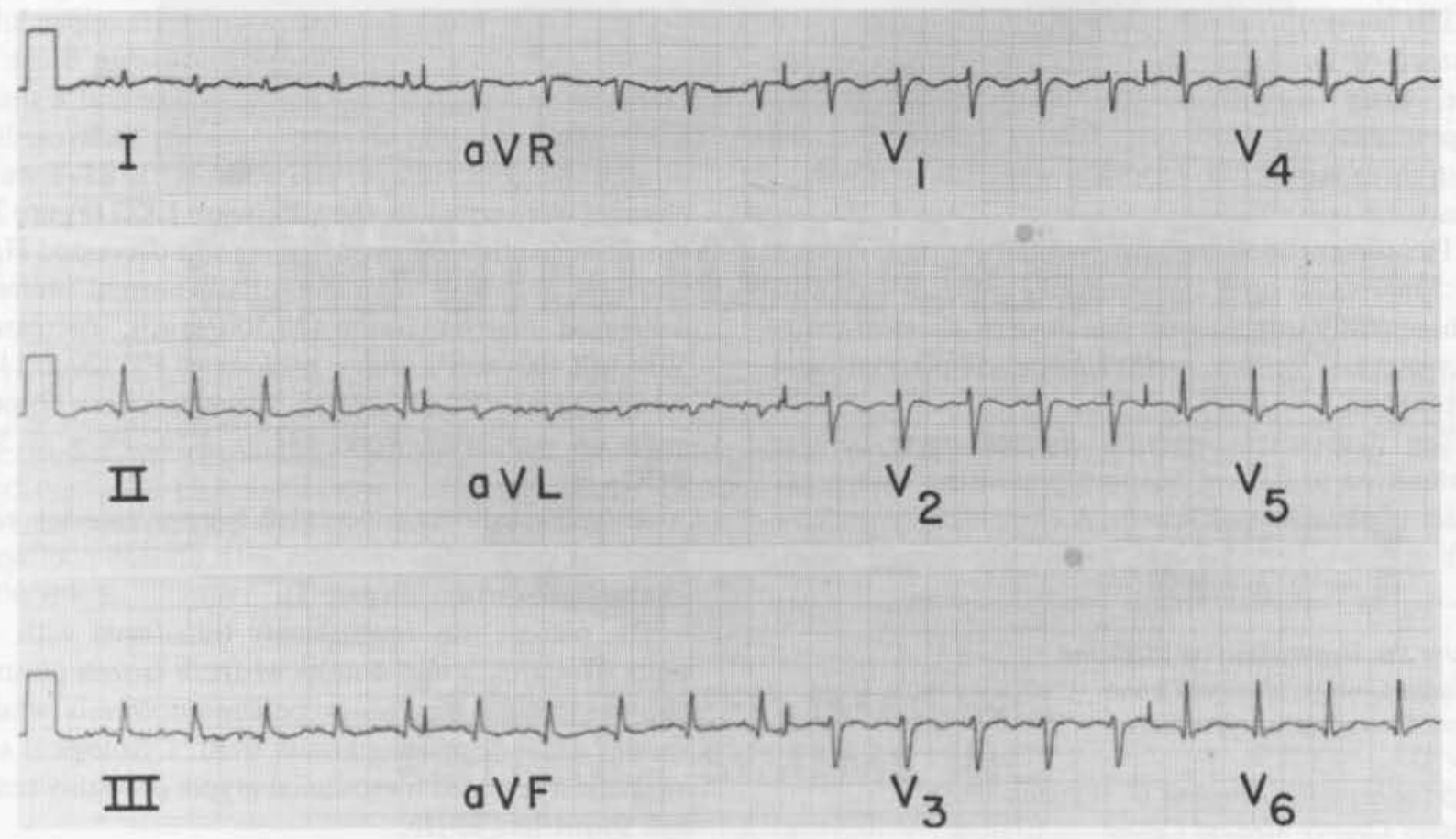

Figure 2. EKG - prior to tap demonstrating sinus tachycardia and borderline low voltage. 


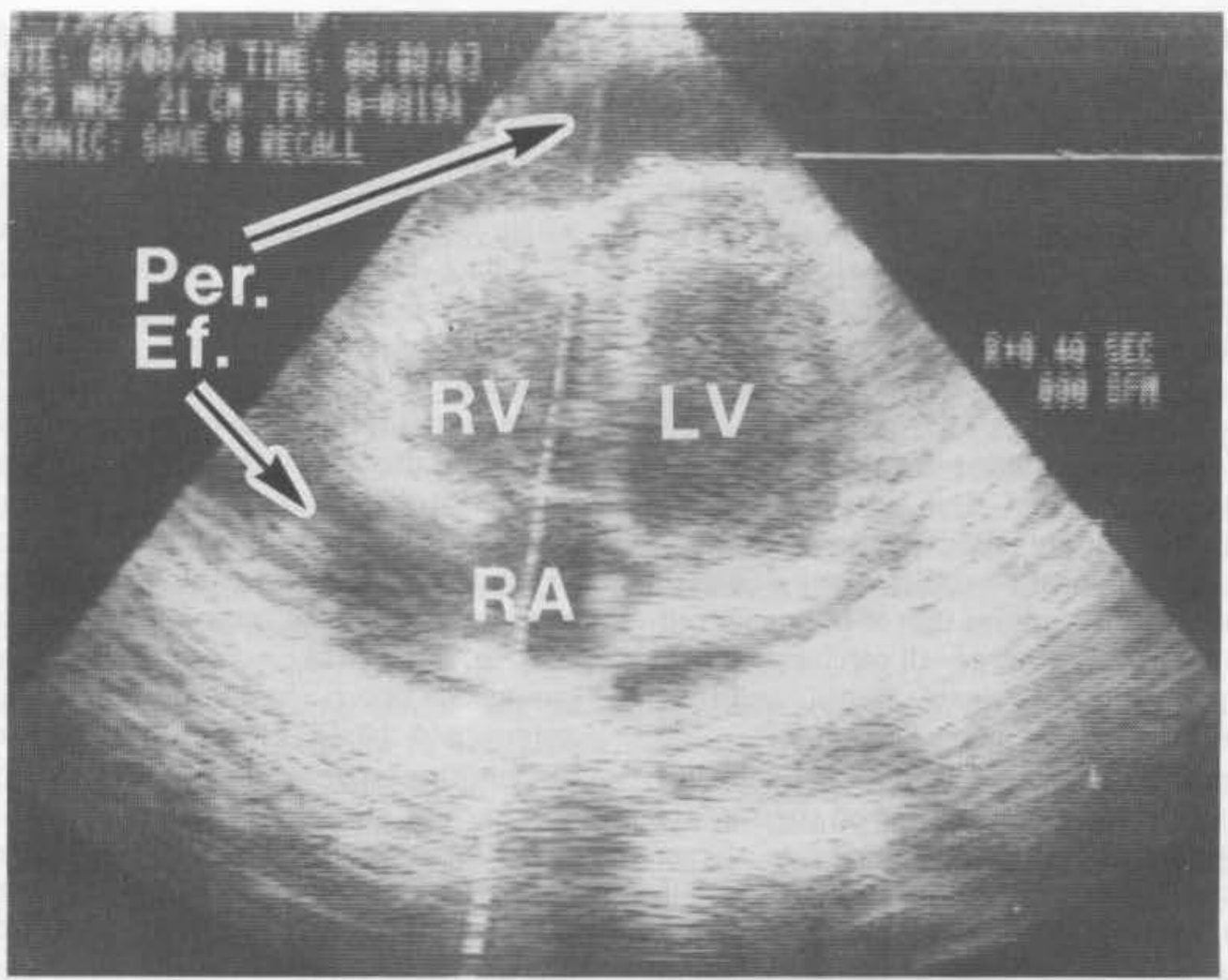

Figure 3: 2-D parasternal long axis echocardiogram demonstrating large anterior and posterior pericardial effusions prior to pericardial tap (RV-right ventricle; LV-left ventricle).

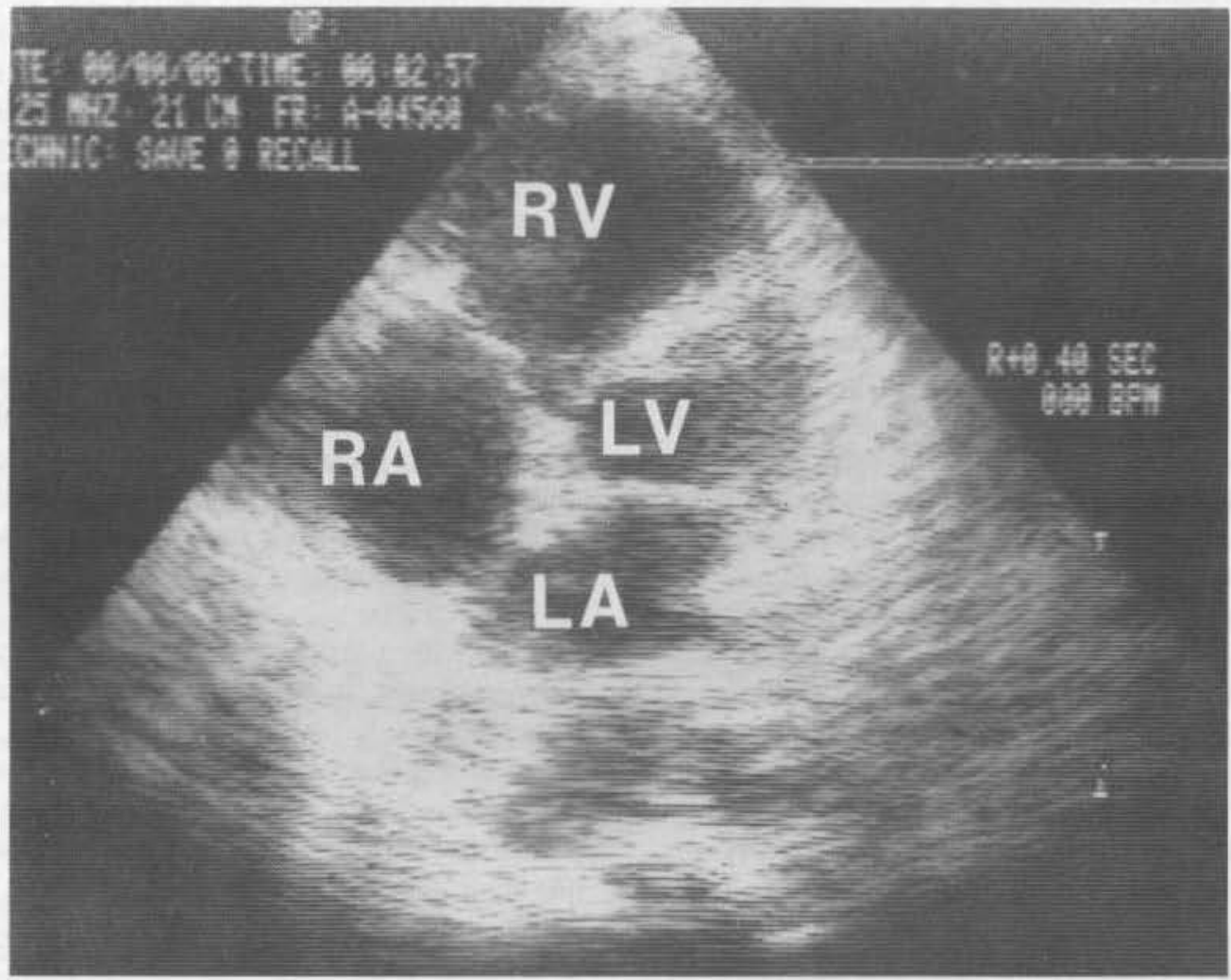

Figure 4: 2-D, 4-chamber apical echocardiogram taken immediately post pericardial tap, revealing no significant pericardial effusion. (RV-right ventricle, RA-right atrium, LR-left ventricle, LA-left atrium). 
the pulsus paradoxicus and neck vein distention, was noted. An echocardiogram revealed marked reduction in the amount of pericardial fluid (Figure 4).

The following day the patient again developed marked shortness of breath and an echocardiogram demonstrated reaccumulation of the pericardial fluid. Repeat pericardiocentesis was performed and a drainage catheter was left in place. Two days later, a subxiphoid limited pericardiotomy was performed.

\section{Discussion}

Large-cell carcinomas comprise $16-20 \%$ of all bronchogenic carcinomas. ${ }^{2}$ The vast majority of large-cell carcinomas are classified as undifferentiated type having abundant cytoplasm with a nuclear pattern distinctly different than that of squamous cell or small-cell carcinomas. Large-cell carcinomas have insignificant amounts of stainable mucin, and thus can be differentiated from adenocarcinomas.

These large-cell carcinomas tend to be large and are peripherally located when viewed on chest $\mathrm{x}$-ray.

According to most series, the overall rate of cardiac metastases in patients with lung cancer is $15-35 \% ;^{3}$ however, in one series cardiac involvement by tumor was noted in $48 \%$ of autopsy-proven cases of bronchogenic carcinomas. ${ }^{4}$

The pericardium is the most common site of metastatic involvement of the heart. In carcinomas of the lung it appears to take place predominantly through lymphatic spread (lymphatic permeation). ${ }^{s}$

Large-cell carcinoma and adenocarcinoma are the histologic cell types of lung cancer with greatest propensity toward cardiac involvement. In a series of 114 cases with autopsy-proven lung cancer,' 10 of 17 patients $(59 \%)$ with large-cell carcinoma had cardiac involvement. This was not significantly different than the $41 \%$ of patients ( 14 of 34 ) with adenocarcinoma having cardiac metastatic disease. Neoplastic involvement of the heart was noted in $21 \%$ of patients ( 8 of 38 ) with squamous cell carcinoma and $24 \%$ of patients ( 6 of 25 ) with small-cell carcinoma. This difference in incidence between patients with large-cell carcinoma campared with squamous cell or small-cell carcinoma was statistically significant.

The diagnosis of cardiac metastases is made in less than $10 \%$ of cases prior to death. ${ }^{6}$ The signs and symptoms of pericardial involvement by malignancy may be subtle and the diagnosis of secondary malgnant lesions of the pericardium may be difficult to make prior to the development of cardiac tamponade. This may occur because the patient's symptoms may be attributed to the underlying carcinomatous process itself. In most cases, the diagnosis will be made only if there is a high index of suspicion and if the patient is examined frequently.

The development of cardiac tamponade depends to a great extent upon the rate at which pericardial fluid accumulates. ' Normally, the pericardial sac contains less than $50 \mathrm{ml}$ of fluid and the mean intrapericardial pressure is close to 0 (atmospheric pressure). Additional volume accumulation of up to 1 to 2 liters of fluid is well tolerated, with little change in intracardial pressure if it occurs slowly, since elastic fibers within the pericardium allow gradual distension to take place. However, with the rapid accumulation of greater than 150 to $200 \mathrm{ml}$ of fluid (or less if the pericardium is thick and noncompliant from neoplastic involvement) there is less time for stretchig to occur and a steep rise in intrapericardial pressure results. As pericardial fluid continues to accumulate, intrapericardial pressure rises further, and right and left ventricular diastolic pressures increase as well. Eventually intrapericardial and ventricular diastolic pressures equilibrate, transmural distending pressure falls to zero, stroke volume declines, and cardiac tamponade occurs. In an effort to maintain cardiac output and blood pressure several compensatory mechanisms occur including a reflex tachycardia, increase in ejection fraction, and arteriolar vasoconstriction. With continued cardiac tamponade, these compensatory mechanisms fail and perfusion to vital organs decreases leading to circulatory collapse, electrical and mechanical dissociation, and death.

The diagnosis of neoplastic cardiac tamponade should be suspencted in a patient with a known malignancy who presents with one of more of the following symptoms:

1) Dyspnea

2) Extreme anxiety or apprehension

3) Precordial oppressive pain aggravated by lying down, coughing or taking deep breaths and improved after sitting up and leaning forward

4) Syncope

Rarely, cardiac tamponade maybe the first presenting sign of a malignancy.$^{s-10}$

Once the possibility of cardiac tamponade is considered, careful physical examination should help confirm the diagnosis. Common findings on examination include:

1) Hypotension with a narrow pulse pressure

2) Pulsus paradoxicus

3) Tachycardia

4) JVD with a prominent systolic $X$ descent.

Interestingly, a pericardial friction rub is rarely detected.

A normal heart size on chest $\mathrm{x}$-ray does not rule out the possibility of a hemodynamically significant pericardial effusion since the cardiac silhouette will not enlarge unless at least $250 \mathrm{ml}$ of fluid are present within the pericardial space.? With large pericardial effusions the heart assumes a globular or "water bottle" configuration. The appearance of electrical alternans on the ECG often indicates the development of cardiac tamponade, particularly when it involves the $\mathrm{P}$ wave as well as the $\mathrm{QRS}-\mathrm{T}$ complex. This 
phenomenon is felt to represent the pendulum-like motion of the heart within the pericardial sac. ${ }^{11}$

Echocardiography has been shown to be a sensitive and accurate procedure for detecting pericardial effusion. Findings consistent with cardiac tamponade include an enhanced augmentation of the normal inspiratory increase in right ventgricular size and inspiratory decrease in left ventricular size, ${ }^{12}$ a decrease in the $\mathrm{E}$ - F slope of the mitral value, ${ }^{12}$ early diastolic inward buckling of the right atrial wall ${ }^{13}$ and diastolic collapse of the right ventricle. ${ }^{14}$

Cardiac catheterization classically reveals elevation and equalization of intrapericardial, right atrial, right and left ventricular diastolic, and pulmonary artery wedge pressures. Right atrial pressure tracings show a prominent systolic $\mathrm{X}$ descent and a decreased or absent early diastolic $\mathrm{Y}$ descent.

After the diagnosis of cardiac tamponade is established, it is essential that treatment be instituted without delay. Circulatory support with intravascular volume expansion should be administered while the patient is being prepared for pericardiocentesis. This procedure is then performed with the patient's head and thorax tilted up so that the pericardial fluid accumulates in an anterior and inferior position. The preferred site for pericardiocentesis is the angle between the xiphoid process and the left costal arch (subxiphoid approach) since it is extrapleural and avoids the major epicardial coronary and internal mammory arteries. ${ }^{7}$

The aspirating needle (with its metal hub attached by a sterile connector to the V lead of an ECG machine) is then directed posteriorly at a 15-degree angle towards the right sternoclavicular joint or left shoulder. Continuous ECG monitoring during the procedure is essential. The needle is advanced until pericardial fluid is obtained or ST segment elevation (indicating a current of injury as the epicardial surface of the right ventricle has been contacted) is seen on the ECG. If ST segment elevation is observed the needle is slowly withdrawn until pericardal fluid is aspirated. After the removal of the first 50 to $100 \mathrm{ml}$ of fluid, clinical improvement with alleviation of shortness of breath, elevated arterial blood pressure and disappearance of pulsus paradoxicus is often observed. Approximately $1 / 3$ of malignant pericardial effusion are serous and the remaining $2 / 3$ are serosanginous or hemorrhagic. ${ }^{6}$ Cytological examination of pericardial fluid is positive for malignancy in approximately $75-90 \%$ of cases. ${ }^{10,15,16,17}$

Following successful pericardiocentesis, there is a tendency for malignant effusions to reform and cardiac tamponade to recurr. Therefore, additional treatment is often required. Surgical drainage procedures such as a subxiphoid pericardiotomy or creation of a pericardial pleural window should be considered for rapidly reaccumulating effusions. ${ }^{18,19}$
Both procedures are better tolerated with less morbidity and mortality than pericardiectomy (the preferred treatment for constrictive pericarditis) which is a major surgical procedure that may not be justifiable in a critically ill debilitated patient with terminal disease and a poor prognosis.

Another approach that may be used in controlling recurrent malignant pericardial effusions is the intrapericardial administration of sclerosing agents (chemotherapeutic agents or radioactive isotopes) following pericardial catheter drainage. ${ }^{18,19}$ The agents induce an inflammatory response resulting in obliteration of the pericardial space. Radiotherapy is another treatment modality that should be considered in patients with radiosensitive tumors. ${ }^{18,19}$

\section{References.}

1. Silverberg E, Lubera JA: Cancer statistics, 1988. CA 1988;38(1):5-22.

2. Yesner R, Carter D: Pathology of carcinoma of the lung. Clin Chest Med 1982;3(2):257-288.

3. Strauss B, Matthews M, Cohen M, Simon R, Tegada F: Cardiac metastases in lung cancer. Chest 1977;71:607-611.

4. Sobel M, Rodman T, Pastor B: The incidence and clinical significance of cardiac involvement in bronchogenic carcinoma. Amer J Med Sci 1960;240:739-748.

5. Onuigbo, WIB: The spread of lung cancer to the heart, pericardium and great vessels. Jpn Heart J 1974;15:234-238.

6. Cohen JL: Neoplastic pericarditis. Cardiovasc Clin 1976;7(3):257-269.

7. Lorell BH, Braunwald E: Pericardial disease. In Braunwald E. (ed): Heart Disease, A Textbook of Cardiovascular Medicine, Philadelphia, W.B. Saunders Co., 1988:pp 1484-1534.

8. Damuth TE, Bush CA, Leier CV: Adenocarcinoma of the lung; Pericardial tamponade as the major presenting feature. Ohio State Med J 1979;75:25-27.

9. Fraser RS, Viloria JB, Wang NS: Cardiac tamponade as a presentation of extra cardiac malignancy. Cancer 1980;45:1697-1704.

10. Haskell RJ, French WJ: Cardiac tamponade as the initial presentation of malignancy. Chest 1985;88:70-73.

11. Usher B, Popp R: Electrical alternans: Mechanisms in pericardial effusion. Am Heart J 1972;83:459-463.

12. D'Cruz IA, Cohen HC, Prabhu R, Glick G. Diagnosis of cardiac tamponade by echocardiography. Circulation 1975;52:460-465.

13. Kronzon I, Cohen M, Winer H: Diastolic atrial compression: A sensitive echocardiographic sign of cardiac tamponade. JACC 1983;2:770-775.

14. Armstrong, WF, Schilt BF, Helper DJ, Dillon JC, Feigenbaum H: Diastolic collapse of the 
right ventricle with cardiac tamponade: An echocardiographic study. Circulation 1982;65:1491-1496.

15. Zipf RE, Johnston WW: The role of cytology in the evaluation of pericardial effusions. Chest 1972;62:593-596.

16. Posner MR, Cohen GI, Skarin AT: Pericardial disease in patients with cancer. Am J Med 1981;71:407-413.
17. Lopez JM, Delgado JL, Tovar E, Gonzalez AG. Massive pericardial effusion produced by extracardiac malignant neoplasms. Arch Intern Med 1983;143:1815-1816.

18. Theologides A: Neoplastic cardiac tamponade. Semin Oncol 1978;5:181-192.

19. McKenna Jr RJ, Ali MK, Ewer MS, Frazier OH: Pleural and pericardial effusions in cancer patients. Curr Probl Cancer 1985;9(6):1-44. 